\title{
Ferroportin1 is required for normal iron cycling in zebrafish
}

\author{
Paula G. Fraenkel,1,2 David Traver,1 Adriana Donovan, ${ }^{1}$ David Zahrieh, ${ }^{3}$ and Leonard I. Zon' \\ 1Division of Hematology/Oncology, Children's Hospital, Karp Research Laboratories, Boston, Massachusetts, USA. \\ 2Division of Hematology/Oncology, Beth Israel Deaconess Medical Center, Boston, Massachusetts, USA. \\ ${ }^{3}$ Department of Biostatistics and Computational Biology, Dana-Farber Cancer Institute, Boston, Massachusetts, USA.
}

\begin{abstract}
Missense mutations in ferroportin1 (fpn1), an intestinal and macrophage iron exporter, have been identified between transmembrane helices 3 and 4 in the zebrafish anemia mutant weissherbst $\left(\right.$ web $^{\left.\mathrm{Tp} 85 \mathrm{c}^{-/-}\right) \text {and in }}$ patients with type 4 hemochromatosis. To explore the effects of fpn1 mutation on blood development and iron homeostasis in the adult zebrafish, $\mathrm{web}^{\mathrm{Tp} 85 \mathrm{c}^{-/-}}$zebrafish were rescued by injection with iron dextran and studied in comparison with injected and uninjected WT zebrafish and heterozygotes. Although iron deposition was observed in all iron-injected fish, only $\mathrm{web}^{\mathrm{TP} 85 \mathrm{c}^{-/-}}$zebrafish exhibited iron accumulation in the intestinal epithelium compatible with a block in iron export. Iron injections initially reversed the ane-

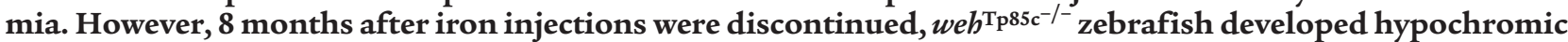
anemia and impaired erythroid maturation despite the persistence of iron-loaded macrophages and elevated hepatic nonheme iron stores. Quantitative real-time RT-PCR revealed a significant decrease in mean hepatic transcript levels of the secreted iron-regulator bepcidin and increased intestinal expression of $f p n 1$ in anemic $w_{e b}{ }^{\mathrm{T}} 85 c^{-/-}$adults. Injection of iron dextran into WT or mutant zebrafish embryos, however, resulted in significant increases in hepcidin expression 18 hours after injection, demonstrating that hepcidin expression in zebrafish is iron responsive and independent of fpn1's function as an iron exporter.
\end{abstract}

\section{Introduction}

According to a current model of iron transport (1), duodenal enterocytes and placental syncytiotrophoblasts are the principle cells responsible for iron acquisition in mammals. At the apical surface of the duodenal enterocyte, the divalent metal transporter 1 (DMT1) mediates uptake of iron from the intestinal lumen into the enterocyte $(2,3)$. Absorbed iron may be stored bound to ferritin or exported across the enterocyte's basolateral membrane to the circulation via ferroportin1 (fpn1) (4). In the plasma, iron binds transferrin and is subsequently imported into erythroid precursors and other cells via transferrin-receptor-mediated endocytosis. Erythrocytes have no known means of iron export but rather incorporate iron into hemoglobin. Macrophages serve as iron scavengers, phagocytosing senescent erythrocytes, degrading hemoglobin, and storing iron incorporated in ferritin.

Hepcidin, a small cysteine-rich peptide with antimicrobial properties $(5,6)$, has recently been identified as a key regulator of iron absorption and utilization. Produced in the liver, hepcidin circulates in plasma and is excreted in the urine. Hepcidin protein levels appear to be largely regulated by transcriptional control (1). Hepcidin null mice develop iron overload (7) while animals that overexpress hepcidin develop severe hypochromic anemia (8). Hepatic expression of the hepcidin gene decreases in response to iron deficiency, hypoxia, and anemia (9) while expression increases in response to iron overload $(10)$ or inflammation $(9,11)$.

Nonstandard abbreviations used: $\mathrm{CH}$, cellular hemoglobin; DAB, diaminobenzidine; DMT1, divalent metal transporter 1; FSC, forward scatter; fpn1, ferroportin1; HFE, hemochromatosis gene product; IRE, iron regulatory element; MCV, mean corpuscular volume; pcm, polycythemia; SSC, side scatter; eeb $^{\mathrm{Tp} 85 \mathrm{c}^{-/-}}$, zebrafish anemia mutant weissherbst.

Conflict of interest: The authors have declared that no conflict of interest exists.

Citation for this article: J. Clin. Invest. 115:1532-1541 (2005).

doi:10.1172/JCI23780.
The zebrafish, Danio rerio, provides an excellent system for the identification and analysis of genes involved in iron metabolism and erythroid development. Analogous to higher vertebrates, zebrafish exhibit multilineage hematopoiesis, resulting in erythroid, monocyte, granulocyte, and thrombocyte lineages, and undergo hemoglobin switching (12). Large-scale genetic screens for embryonic hypochromic anemia have identified recessive mutations with defects in heme synthesis (13), globin production (12), and iron acquisition via DMT1 (14) and transferrin receptor (15). Positional cloning of the mutation responsible for the hypochromic zebrafish mutant weissherbst (weh) resulted in the identification of the basolateral iron exporter, fpn1, (4) also known as IREG1 (16) and MTP1 (17), a conserved protein with 10 putative transmembrane domains and a functional iron response element (18) in the 5 ' untranslated region.

Two alleles of the weh phenotype have been described previously (19). Both have a homozygous recessive pattern of inheritance. The $w_{e} b^{\text {Th2 } 238}$ allele has a premature stop mutation and is thought to be a null allele (4). The web ${ }^{\mathrm{T} p 85 c}$ allele is of particular interest because it encodes a missense mutation (Leu 167 to Phe) in the same conserved region of fpn1 (4) as several of the missense mutations identified in patients with type 4 hemochromatosis (20-26). Both $w e h^{\mathrm{TP} 85 c}$ and $w e b^{\mathrm{Th} 238}$ homozygotes develop severe anemia by 48 hours after fertilization and die between 10 and 14 days of age. A single intravenous or intramuscular iron dextran injection allowed the homozygote embryos to survive (4) for several weeks but not to reach maturity.

Here we demonstrate that administration of multiple iron injections enabled the $w_{e b} b^{\mathrm{T} p 85 c^{-/}}$zebrafish to survive to adulthood, facilitating evaluation of the effects of the fpn 1 mutation on blood development and iron homeostasis. We provide evidence that $w_{e} h^{\mathrm{T} 85 c^{-/}}$adults have impaired iron export, both from enterocytes and macrophages, and that normal fpn 1 function is not required for iron-responsive regulation of hepcidin in the zebrafish. 
A

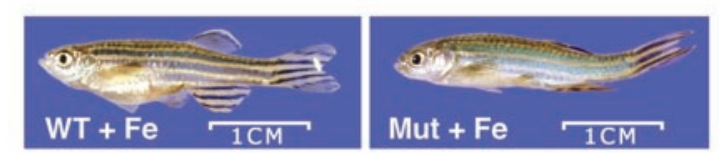

C

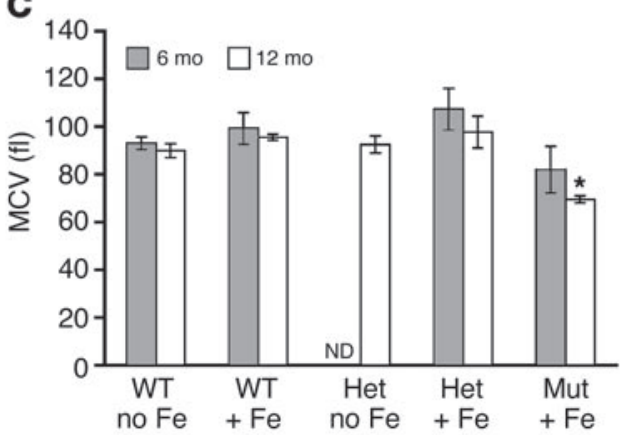

B

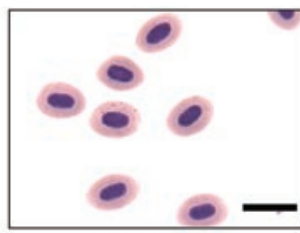

WT no Fe

D

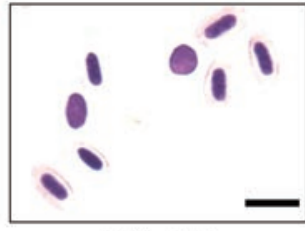

Mut $+\mathrm{Fe}$

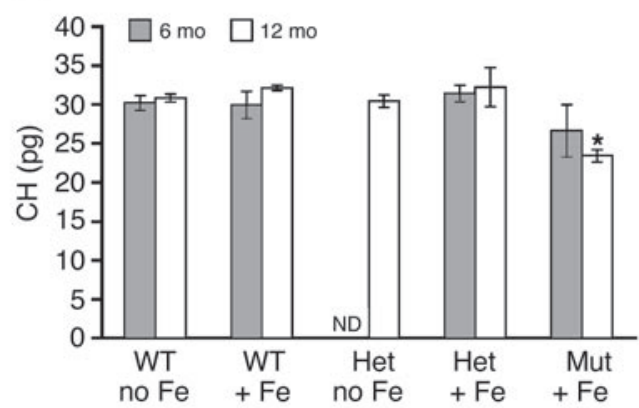

Figure 1

The phenotype of the weh mutation in adult zebrafish. (A) Photographs of an iron-injected WT zebrafish (left) compared with a pale, iron-injected homozygote $\left(w_{e} h^{\top p 85 c^{-1}}\right.$ ) (right) at 1 year of age. (B) Erythrocytes from peripheral blood of iron-injected weh $h^{\text {Tp } 85 c^{-1-}}$ (Mut + Fe; right) at 1 year of age exhibit pale cytoplasm and decondensed nuclei compared with WT zebrafish (WT no Fe; left) stained with Wright-Giemsa. Magnification $\times 100$. Scale bars: 10 microns. (C and D) Erythroid indices were obtained with an ADVIA 120 automated analyzer at 6 months (gray bars, $n=3-5$ ) and 12 months (white bars, $n=4-6$ ) for each cohort: uninjected weh ${ }^{\top p 85 c^{+/+}}$zebrafish (WT no Fe), iron-injected weh ${ }^{\top p 85 c^{+/+}}$zebrafish (WT + Fe),

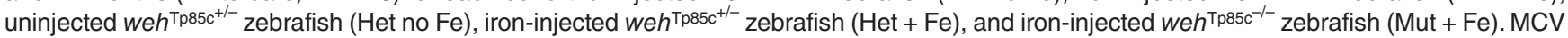
(C) is a measure of erythrocyte size, while $\mathrm{CH}$ (D) quantitates the amount of hemoglobin per erythrocyte. Data shown are means $\pm \mathrm{SE}$. ND, not done. ${ }^{*} P<0.0001$ compared with 12-month-old iron-injected WT zebrafish.

\section{Results}

The effects of a missense mutation in fpn 1 have not been evaluated previously in an adult model organism. In order to study the effects of fpn 1 deficiency on erythroid development and iron homeostasis, we rescued $w e b^{\mathrm{Tp}} 85 \mathrm{c}$ homozygotes to adulthood by administering iron dextran injections early in development. Without iron injections, 0 of 529 homozygous mutants were alive at 3 weeks. Treatment with a single iron injection at 72 hours rescued the anemia phenotype and allowed 47 of 87 (54\%) of the mutants $\left(\right.$ web $\left.^{\mathrm{Tp} 85 c^{-/-}}\right)$ to survive to 1 month, which was similar to the survival rate of ironinjected (37 of $61 ; 61 \%$ ) and uninjected (47 of $89 ; 53 \%$ ) controls $\left(\right.$ we $^{\mathrm{Tp} 85 \mathrm{c}^{+/-}}$and $w_{e} h^{\mathrm{Tp} 85 c^{+/+}}$). Without additional iron injections, the mutant animals did not continue to mature. After a total of 4 injections of iron dextran were administered to the $\mathrm{weh}^{\mathrm{Tp} 85 \mathrm{c}^{-/-}}$zebrafish (day 3 , week 5, week 8, and week 16), the animals reached adult size. This regimen was adopted for subsequent experiments.

As fpn 1 has been shown to be expressed in the yolk syncytial layer that separates the embryo from the iron-rich yolk, we postulated that the weh mutation could have a maternal-dominant effect, impairing export of iron to the embryo from maternally derived stores. Weh ${ }^{\mathrm{Tp} 85 c}$ homozygous females crossed with we $^{\mathrm{Tp} 85 c^{+/+}}$males produced only apparently normal, nonanemic embryos (data not shown). This indicates that iron transfer into the zebrafish embryo does not depend on maternal transcripts of $f p n 1$. Either $f p n 1$ transcripts derived from the zygote are sufficient, or iron is transferred into the embryo via an fpn1-independent mechanism.

The $w_{e} h^{\mathrm{T}} 85 \mathrm{c}$ homozygotes and controls were monitored as they aged. At 6 months of age, 2 months after the last iron injection, $w e b^{T p} 85 c^{-/-}$did not differ in appearance from injected or uninjected controls. By 12 months of age, however, the homozygote animals had developed pallor (Figure 1A), and the peripheral blood was hypochromic on a blood smear (Figure 1B). To assess the severity of anemia, we measured mean corpuscular volume (MCV) (Figure 1C) and cellular hemoglobin (CH) (Figure 1D) from the peripheral blood of individual zebrafish. MCV is a measure of erythrocyte size while $\mathrm{CH}$ indicates the amount of hemoglobin per erythrocyte. At 6 months of age, there was no significant difference in $\mathrm{MCV}$ and $\mathrm{CH}$ among the cohorts. The MCV was $81.8 \pm 9.82 \mathrm{fl}$ for web $^{\mathrm{Tp} 85 c^{-1-}}$ zebrafish versus $99.2 \pm 6.65$ for iron-injected WT zebrafish $(P=0.26)$. CH was $26.6 \pm 3.4 \mathrm{pg}$ for $w_{e} b^{\mathrm{Tp} 85 c^{-/-}}$zebrafish versus $29.9 \pm 1.74 \mathrm{pg}$ for iron-injected WT zebrafish $(P=0.50)$. When the zebrafish reached 12 months of age, however, we observed a reduction in MCV in ironinjected $w e h^{\mathrm{Tp} 85 c^{-/}}$zebrafish compared with iron-injected WT zebrafish $(69.6 \pm 1.43 \mathrm{fl}$ vs. $95.4 \pm 1.36 \mathrm{fl}$, respectively; $P<0.0001)$ or uninjected WT zebrafish $(89.9 \pm 2.93 \mathrm{fl} ; P=0.0008)$. Similarly, the amount of hemoglobin per erythrocyte $(\mathrm{CH})$ was reduced significantly from $32.2 \pm 2.53 \mathrm{pg}$ in iron-injected WT zebrafish to $23.4 \pm 0.78 \mathrm{pg}$ in ironinjected weh $^{\mathrm{Tp} 85 c^{-/-}}$zebrafish $(P<0.0001)$. The decrease observed in both erythrocyte size and hemoglobin content are consistent with anemia due to impaired hemoglobin production.

To examine the effect of fpn 1 deficiency on the adult site of zebrafish hematopoiesis, kidneys from individual 12-month-old weh $^{\mathrm{Tp} 85 c^{-/-}}$zebrafish and controls were dissected. Flow cytometry was used to separate the kidney marrow cells into 4 populations based on differences in forward scatter (FSC) and side scatter (SSC) in controls (Figure 2A) and web $^{\mathrm{Tp} 85 c^{-/-}}$zebrafish (Figure 2B). The mean percentages of cells in these populations were compared (Figure 2C). This revealed a 3 -fold reduction in the mean percentage of mature

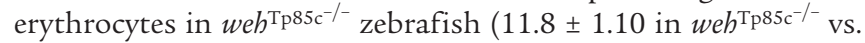
$36.8 \pm 1.93$ in WT plus iron; $P<0.0001)$. Also observed was a doubling 
A

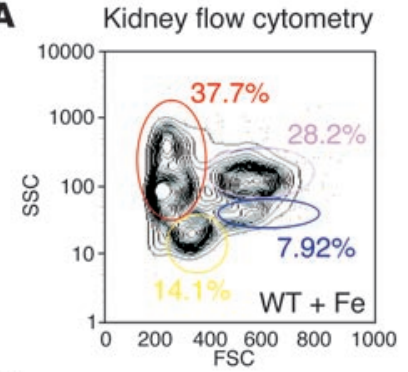

B

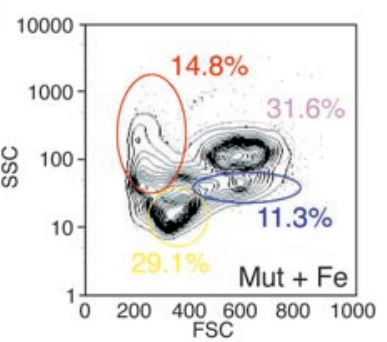

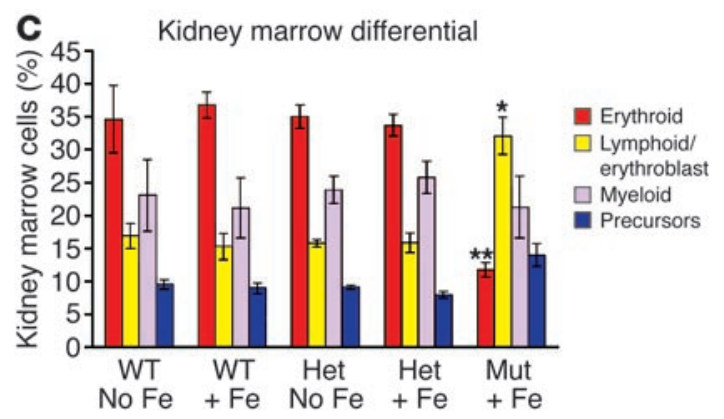

D

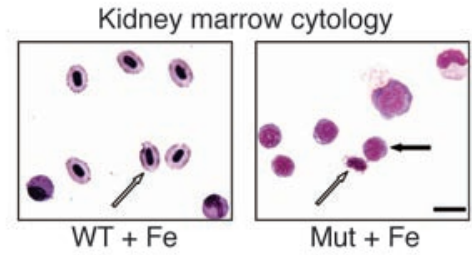

Figure 2

Marrow differentials obtained from the kidneys of adult zebrafish at 1 year of age. From each animal, $1 \times 10^{5}$ kidney marrow cells were analyzed by FSC and SSC, according to a previously defined method (48). Representative flow cytometry plots are shown from an iron-injected WT (A) and a weh $\mathrm{h}^{\mathrm{Tp} 8 \mathrm{c}^{-/-}}$(B) zebrafish. Four major populations were delineated: erythroid (red ellipse), lymphoid/erythroblast (yellow ellipse), myeloid (lilac ellipse), and the most immature precursor cells (blue ellipse). Shown next to each ellipse is the percentage of kidney marrow cells in each gate. (C) Mean percentages of kidney marrow cells in each of the major cell populations; $n=3-5$ per cohort. ${ }^{*} P=0.006 ;{ }^{* *} P<0.0001$ compared with iron-injected WT zebrafish. (D) Kidney marrow cytospins stained with Wright-Giemsa for iron-injected WT (left) compared with iron-injected weh $h^{T p 85 c^{-/-}}$zebrafish (right). Open arrows indicate erythrocytes, while black arrow indicates 1 of 5 erythroblasts shown in the $w^{-h^{T p} 85 c^{--}}$zebrafish photomicrograph. Magnification, $\times 100$. Scale bar: 10 microns.

in the mean percentage of the lymphoid/erythroblast population that includes both lymphoid cells and small erythroblasts $(32.1 \pm 2.84$ in $w^{\mathrm{T}^{\mathrm{T}} 85 \mathrm{c}^{-/-}}$vs. $15.3 \pm 1.96$ in WT plus iron; $P=0.006$ ). Enumeration of manual differentials (Table 1) from cytospin preparations of kidney marrow confirmed a significant increase in the percentage of erythroblasts (Figure 2D) in weh $^{\mathrm{T} p 85 \mathrm{c}^{-/}}$zebrafish compared with that in iron-injected WT zebrafish $(43.8 \pm 6.34$ vs. $9.25 \pm 0.75 ; P=0.02)$ without a significant difference in the percentage of lymphocytes (Table 1). These data indicate that $w^{\mathrm{T}} \mathrm{h}^{\mathrm{p} 85 c^{-/-}}$zebrafish undergo a partial arrest in late erythropoiesis that results in a shift in the marrow population toward erythroblasts.

While fpn 1 has been proposed as the principle enterocyte iron exporter, it has not been demonstrated previously that a missense mutation in fpn1 impairs intestinal iron export. At 6 months, all the iron-rescued we $^{\mathrm{Tp} 85 \mathrm{c}^{-/-}}$zebrafish evaluated $(n=4)$ had strong staining of the intestinal villi consistent with the hypothesized block in iron export caused by fpn 1 deficiency (Figure 3A). This was not observed in any of the other cohorts at 6 months (Table 2). At 12 months, again, only web ${ }^{\mathrm{T}} 85 \mathrm{c}^{-/-}$ homozygotes exhibited nonheme iron staining in the intestinal villi. Although the staining was less intense (Figure 3B), it was apparent with diaminobenzidine (DAB) (Figure 3C) in 3 of the 4 homozygotes, consistent with impaired iron export from the enterocytes.

In the vertebrate marrow, macrophages store iron for release to developing erythroid cells. While it is expected that iron dextran injection would load the reticuloendothelial system with iron, the delayed onset of anemia in iron-injected weh $^{\mathrm{T} P 85 c^{-}-}$zebrafish raises the possibility that kidney marrow iron stores were exhausted by 12 months of age. At 6 months, increased kidney iron stores were evident upon Perls' staining in the macrophages of iron-injected fish, regardless of genotype (Figure 4A). Increased kidney macrophage iron stores were again demon-

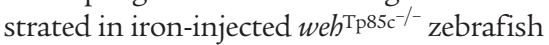
at 12 months (Figure 4, B and C), the same weh $^{\mathrm{Tp} 85 \mathrm{c}^{-1-}}$ zebrafish shown to be anemic by erythroid indices (Figure 1, C and D). Due to the small size of the zebrafish kidney, it was not feasible to quantitate kidney nonheme iron levels; however, enumeration of iron-laden macrophages per high-powered field approximates kidney iron stores. Evaluation of uninjected heterozygotes at 12 months revealed kidney macrophage iron stores similar to those of uninjected WT zebrafish $(0.75 \pm 0.48$ vs. $4.75 \pm 4.75 ; P=0.43)$. At 6 months and 12 months, the mean numbers of iron-laden kidney macrophages per highpowered field in $w_{e h^{\mathrm{TP}} 85 c^{-} /-}$zebrafish were $38.0 \pm 8.0$ and $43.5 \pm 7.12$, respectively (Figure 4D). These values were not significantly different from those for iron-injected WT zebrafish at 6 months ( $48.3 \pm 27.7$; $P=0.79)$ or 12 months $(34.8 \pm 11.9 ; P=0.55)$. These data indicate that the onset of anemia in $w_{e h^{T} \mathrm{p} 85 c^{-}-}$zebrafish was not associated with depletion of kidney macrophage iron stores.

\section{Table 1}

Manual differentials of kidney marrow

\begin{tabular}{lcccccc} 
& \% Myeloid and lymphoid blasts & $\%$ Myelomonocytes & $\%$ Lymphocytes & $\%$ Thrombocytes & $\%$ Erythroblasts & \% Mature erythrocytes \\
WT no Fe & $8.75 \pm 5.25$ & $44.2 \pm 6.75$ & $9.00 \pm 4.00$ & $1.25 \pm 0.75$ & $13.8 \pm 4.25$ & $23.5 \pm 4.50$ \\
WT + Fe & $7.50 \pm 2.00$ & $44.0 \pm 10.0$ & $7.25 \pm 0.25$ & $1.00 \pm 0.50$ & $9.25 \pm 0.75$ & $30.8 \pm 12.2$ \\
Het no Fe & $3.00 \pm 0.50$ & $27.8 \pm 3.25$ & $13.2 \pm 5.25$ & $1.00 \pm 1.00$ & $15.2 \pm 4.25$ & $38.8 \pm 3.25$ \\
He + Fe & $8.33 \pm 0.60$ & $38.3 \pm 2.52$ & $8.00 \pm 0.50$ & $2.83 \pm 0.93$ & $15.7 \pm 5.50$ & $26.3 \pm 3.48$ \\
Mut + Fe & $5.67 \pm 1.74$ & $32.5 \pm 4.36$ & $8.33 \pm 0.60$ & $0.33 \pm 0.33$ & $43.8 \pm 6.34$ A & $9.33 \pm 0.44 \mathrm{~B}$ \\
\hline
\end{tabular}

Manual differentials obtained from cytospins of kidney marrow from individual 12-month-old zebrafish; $n=2-3$ per cohort. Slides were stained with WrightGiemsa, and 200 cells were counted per zebrafish. Data shown are percentages \pm SE. ANOVA reached statistical significance only for proerythrocyte and mature erythrocyte categories. ${ }^{A} P<0.05$ compared with each of the other cohorts. ${ }^{B} P<0.05$ compared with uninjected WT, uninjected heterozygotes, and iron-injected heterozygotes. 


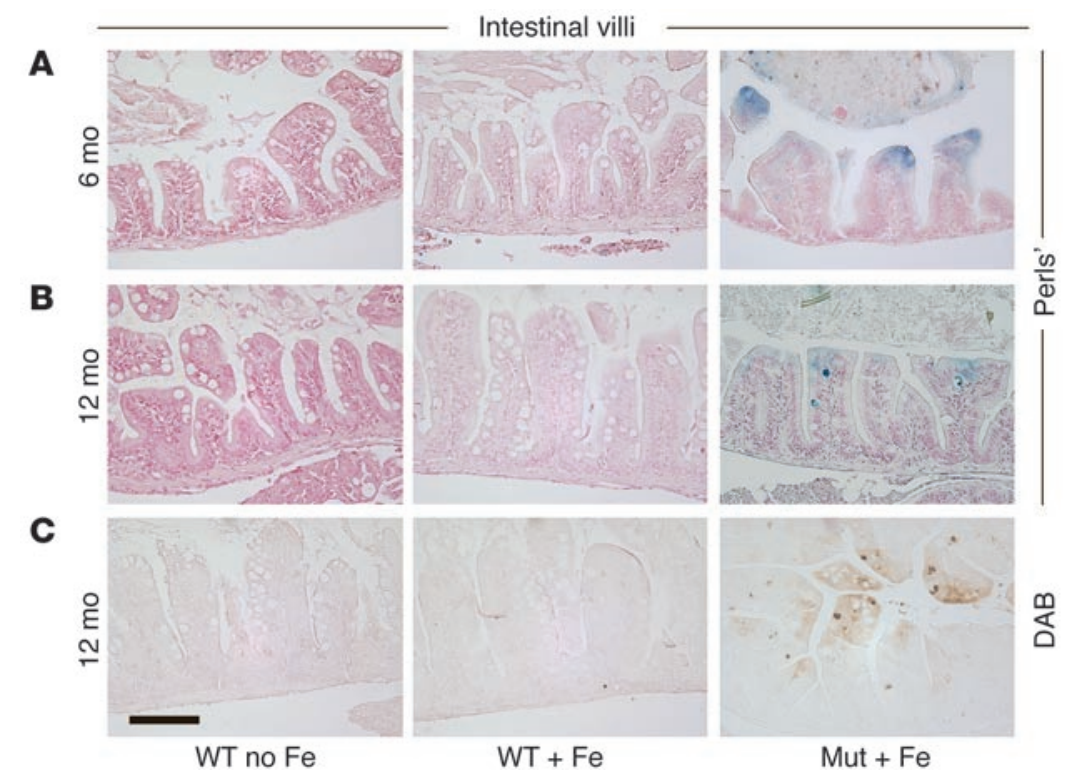

Figure 3

Histology of intestinal villi in uninjected WT (left), WT injected with iron (center), and $w_{e} h^{T p 85 c^{-l}}$ zebrafish injected with iron (right). Perls' stain performed at 6 months of age (A) and 12 months of age (B) revealed nonheme iron accumulation in the intestinal villi of weh $^{\top \mathrm{p} 85 \mathrm{c}^{-/}}$zebrafish but not in controls. Magnification, $\times 40$. Scale bar: 100 microns. (C) DAB-enhanced Perls' staining for nonheme iron at 12 months for the same specimens as shown in $\mathbf{B}$.

Liver iron stores were also increased among the iron-injected fish. At 6 months (Figure 5A), nonheme iron deposition was evident on Perls' staining both diffusely in hepatocytes and localized to Kupffer cells in the iron-injected fish (Table 2). At 12 months, histologic evidence of iron accumulation was still apparent in some of the $\mathrm{weh}^{\mathrm{Tp} 85 \mathrm{c}^{-/-}}$zebrafish (Figure 5B), primarily in Kupffer cells (Figure 5C), and diffuse staining of hepatocytes was absent. In contrast, iron-injected WT zebrafish continued to exhibit diffuse hepatocyte iron staining (Figure 5B) with less Kupffer cell iron staining (Figure 5C). These findings suggest that fpn 1 deficiency impaired iron export from Kupffer cells to a greater extent than it impaired iron export from hepatocytes.

Quantitation of nonheme iron stores using the bathophenanthroline sulfonate chromagenic assay confirmed that iron-injected $w_{e b^{T} \mathrm{p} c^{-/-}}$zebrafish had increased hepatic iron stores. Because the assay required pooling of large numbers of fish, it was initially performed on 3 cohorts at 8 months of age ( $n=1$ pool per cohort), prior to the onset of anemia. Hepatic iron levels at 8 months were elevated in iron-injected WT $(508 \mu \mathrm{g} / \mathrm{g})$ and web $^{\mathrm{Tp} 85 c^{-/-}}$zebrafish $(250 \mu \mathrm{g} / \mathrm{g})$ compared with levels in uninjected WT zebrafish $(40.0 \mu \mathrm{g} / \mathrm{g})$. At 12 months of age, hepatic iron stores were assayed in all cohorts; $n=2-4$ pools per cohort (Figure 5D). Hepatic iron levels in web $^{\mathrm{Tp} 85 c^{-/-}}$zebrafish were significantly greater than those in uninjected WT zebrafish $(111 \pm 29.8 \mu \mathrm{g} / \mathrm{g} v \mathrm{~s} .27 .4 \pm 8.44 \mu \mathrm{g} / \mathrm{g} ; P=0.02)$ but not significantly different from iron levels in iron-injected WT zebrafish $(226 \pm 69.8 \mu \mathrm{g} / \mathrm{g}$; $P=0.20)$. These results indicate that the anemia in $\mathrm{web}^{\mathrm{Tp} 85 \mathrm{c}^{-/-}} \mathrm{zebra}-$ fish was not associated with exhaustion of liver iron stores.

While anemia would be expected to suppress expression of the iron regulator hepcidin and increase $f p n 1$ expression, accumulation of iron in $\mathrm{web}^{\mathrm{Tp} 85 \mathrm{c}^{-/-}}$zebrafish could lead to the opposite effect. To quantitate changes in gene expression, RNA was extracted from the livers and intestines of zebrafish from mutant and control cohorts at 10-12 months of age and used to generate cDNA templates for multiplex realtime PCR amplifying $\beta$-actin and the gene of interest. Transcript abundance, normalized to $\beta$-actin expression, is expressed as a fold increase over a calibrator sample. Although the mean hepatic transcript levels for transferrin (Figure 6A) and $f p n 1$ (Figure 6B) were not significantly different among the mutant and control cohorts, $\mathrm{web}^{\mathrm{Tp} 85 \mathrm{c}^{-/-}}$zebrafish displayed significantly increased expression of intestinal $f p n 1$ (Figure 6C). The mean intestinal transcript level of $f p n 1$ in weh was $8.34 \pm 2.43$, compared with $1.36 \pm 0.46$ in iron-injected WT zebrafish $(P=0.01)$. In situ hybridizations for $f p n 1$ performed on paraffin-embedded sections of zebrafish intestine at 1 year (Figure 7 ) confirmed that the majority of $w_{e b^{\mathrm{Tp} 85 c^{-/}-}}$zebrafish (3/4) had increased intestinal expression of $f p n 1$ that was not apparent in iron-injected WT zebrafish.

Although upregulation of $f p n 1$ in the $w_{e b^{T p} 85 c^{-/-}}$ zebrafish intestine may be related to the local effect of iron accumulation in the enterocyte, downregulation of hepcidin would be consistent with a systemic response to anemia. In $w_{e h^{T}}{ }^{\mathrm{p}^{2} c^{-/-}}$zebrafish, the mean bepcidin transcript level (Figure 6D) was lower than in every other cohort $(0.039 \pm 0.013$ vs. $3.71 \pm 1.17$ in iron-injected WT; $P<0.0001)$. Although hepcidin expression was highly variable among both injected and uninjected controls, there was no significant difference in mean hepcidin transcript levels among the control cohorts, suggesting that the acute effects of iron injection had resolved months after the last treatment.

Zebrafish embryos from 48-66 hours after fertilization exhibit low levels of endogenous hepcidin transcripts (Figure 6E), facilitating the evaluation of factors affecting hepcidin expression. To test the hypothesis that hepcidin is an iron-responsive gene in the zebrafish, embryos at 48 hours after fertilization were injected with iron dextran. The multiplex PCR assay was used to quantitate transcript abundance at specific time points for injected embryos and age-matched controls. An initial small increase in hepcidin transcript levels was observed 2 hours after iron injection $(1.37 \pm 0.37$ vs. $0.003 \pm 0.001$ in age-matched,

\section{Table 2}

Nonheme iron staining

\begin{tabular}{lcccccc}
\hline & \multicolumn{2}{c}{$\begin{array}{c}\text { Iron-Iaden } \\
\text { enterocytes }\end{array}$} & \multicolumn{2}{c}{$\begin{array}{c}\text { Iron-Iaden } \\
\text { hepatocytes }\end{array}$} & \multicolumn{2}{c}{$\begin{array}{c}\text { Iron-Iaden } \\
\text { Kupffer cells }\end{array}$} \\
& $6 \mathrm{mo}$ & $12 \mathrm{mo}$ & $6 \mathrm{mo}$ & $12 \mathrm{mo}$ & $6 \mathrm{mo}$ & $12 \mathrm{mo}$ \\
$\mathrm{WT}$ no Fe & $0 / 4$ & $0 / 4$ & $0 / 4$ & $0 / 4$ & $0 / 2$ & $0 / 4$ \\
$\mathrm{WT}+\mathrm{Fe}$ & $0 / 3$ & $0 / 4$ & $3 / 3$ & $4 / 4$ & $0 / 3$ & $0 / 4$ \\
Het no Fe & $\mathrm{ND}$ & $0 / 4$ & $\mathrm{ND}$ & $0 / 4$ & $\mathrm{ND}$ & $0 / 4$ \\
Het + Fe & $0 / 3$ & $0 / 4$ & $2 / 3$ & $2 / 4$ & $1 / 3$ & $0 / 4$ \\
Mut + Fe & $3 / 3$ & $3 / 4$ & $2 / 3$ & $0 / 4$ & $1 / 3$ & $2 / 4$ \\
\hline
\end{tabular}

Summary of intestine and liver nonheme iron-staining data at 6 months and 12 months of age. Shown in the numerator is the number of individuals in each cohort with increased nonheme iron staining in enterocytes (left) or hepatocytes (center) or more than 10 iron-laden Kupffer cells per high-powered field (right). The denominator is the total number of individuals evaluated. 

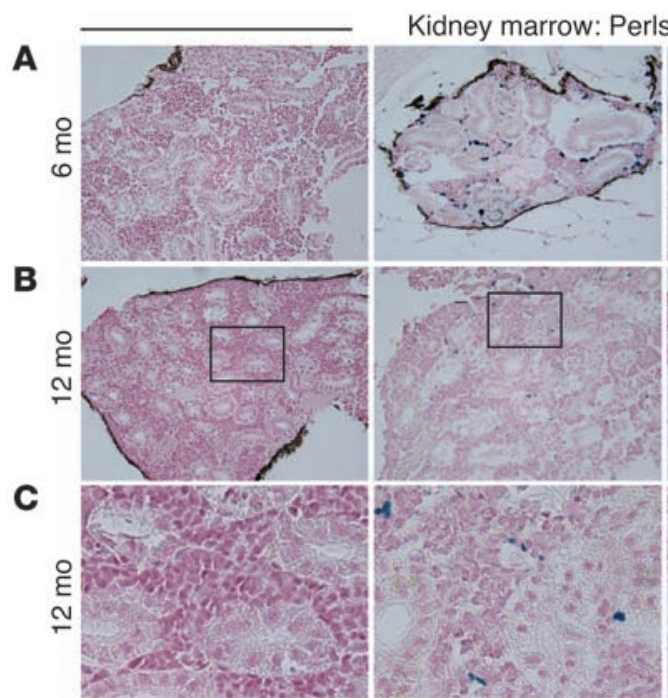

$\mathrm{WT}+\mathrm{Fe}$
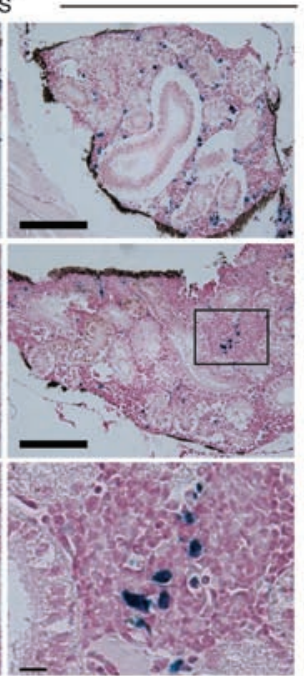

$\mathrm{Mut}+\mathrm{Fe}$

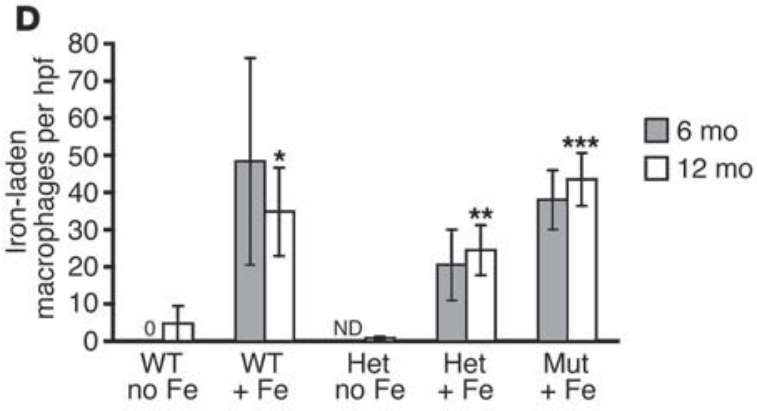

Figure 4

Kidney marrow iron stores. Iron-laden macrophages demonstrated by Perls' staining in uninjected WT (left), WT treated with iron (center), and weh ${ }^{\top p 85 c^{-1-}}$ zebrafish treated with iron (right) that were sacrificed at ages 6 months (A) and 12 months (B). Magnification, $\times 40$. Scale bar: 100 microns. Iron-laden macrophages were located in the hematopoietic tissue that surrounds the pale staining renal tubules. (C) Higher magnification of area marked by rectangle in B. Magnification, $\times 160$. Scale bar: 10 microns. (D) Average number of iron-laden macrophages per high-powered field (hpf) in each of the cohorts at 6 months (gray bars; $n=2-3$ ) and 12 months (white bars; $n=4$ ) of age. Zero indicates that no iron-laden macrophages were identified. ${ }^{\star} P=0.029$ compared with uninjected heterozygotes at 12 months; ${ }^{* \star} P=0.013$ compared with uninjected heterozygotes at 12 months; ${ }^{* \star} P=0.001$ compared with uninjected heterozygotes at 12 months and $P=0.004$ compared with uninjected WT zebrafish at 12 months.

uninjected; $P=0.01)$ followed by a subsequent larger increase 18 hours after iron injection ( $13.0 \pm 5.44$ vs. $0.08 \pm 0.06$ in age-matched, uninjected; $P=0.03$ ). To assess the effects of fpn 1 function on hepcidin expression, transcript levels were measured in weh homozygotes 18 hours after iron dextran injection. Hepcidin expression increased significantly (Figure 6F) in the iron-treated mutants $(14.4 \pm 1.70$ vs. $0.48 \pm 0.17$ in age-matched, uninjected mutants; $P=0.002$ ) and was not significantly different from the level observed in ironinjected WT siblings $(27.9 \pm 7.76)$. This indicates that induction of hepcidin expression does not require normal fpn1 function.
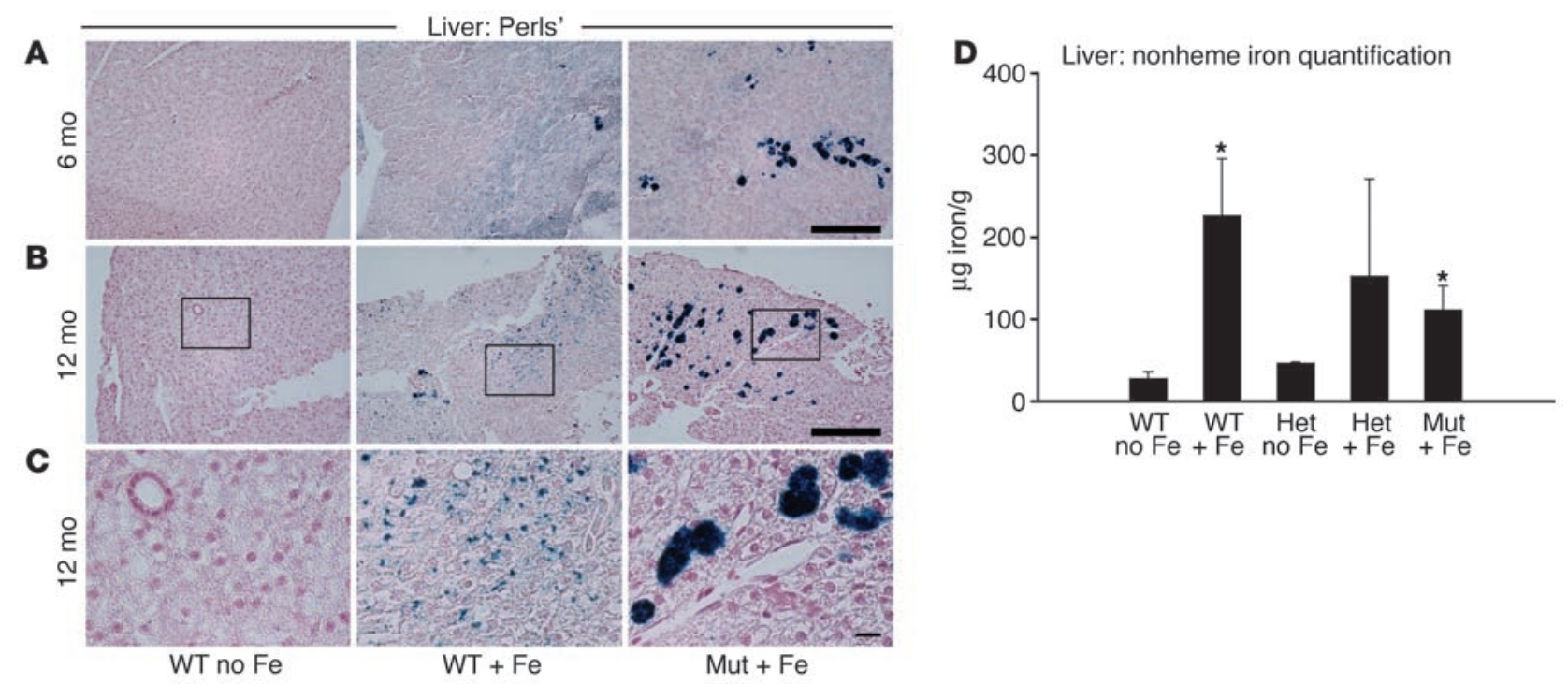

Figure 5

Liver iron stores. Perls' staining demonstrating accumulation of nonheme iron in the liver of uninjected WT (left), iron-injected WT (center), ironinjected weh $^{T p 85 c^{-/-}}$zebrafish (right) at 6 months of age (A) and 12 months of age (B). Magnification, $\times 40$. Scale bar: 100 microns. (C) Higher magnification of area marked by rectangle in B. Magnification, $\times 160$. Scale bar: 10 microns. (D) Nonheme iron levels were quantified ( $\mu g$ iron/g liver) using the bathophenanthroline sulfonate chromagenic assay. Due to the small size of the organs, livers were dissected from individual zebrafish at 12 months of age and pooled according to cohort; $n=2-4$ pooled samples per cohort. ${ }^{*} P=0.02$ compared with uninjected WT zebrafish. 

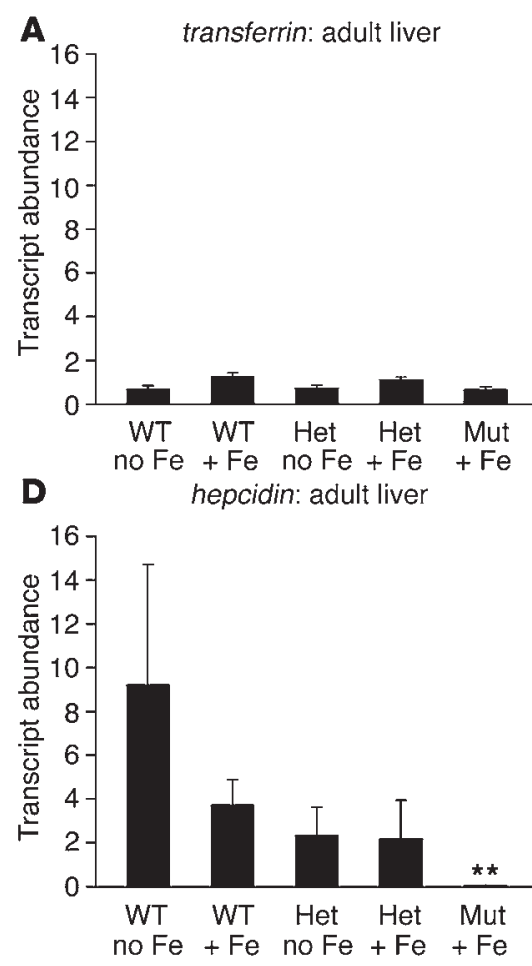

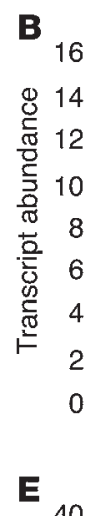

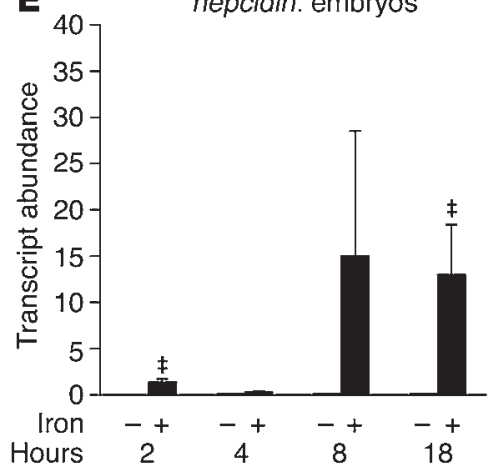

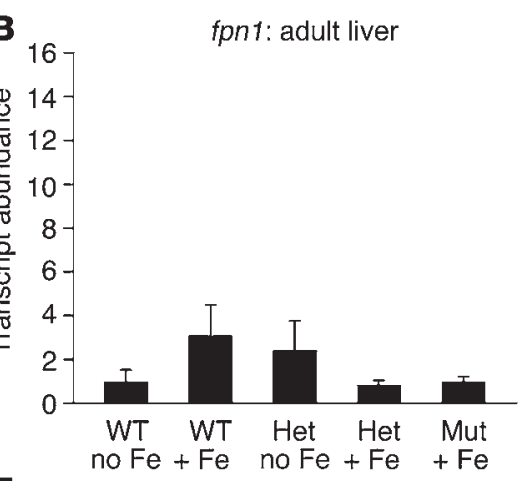

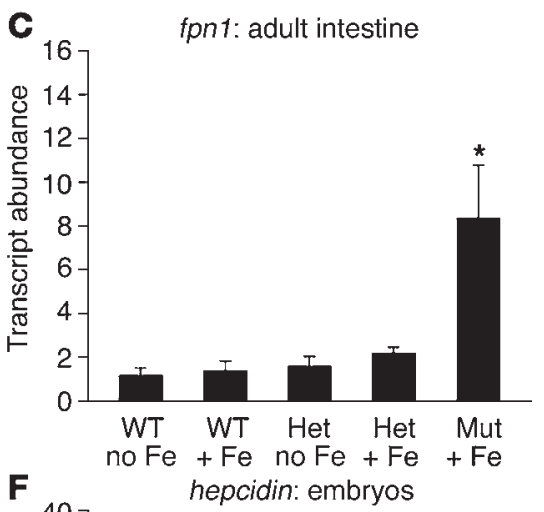

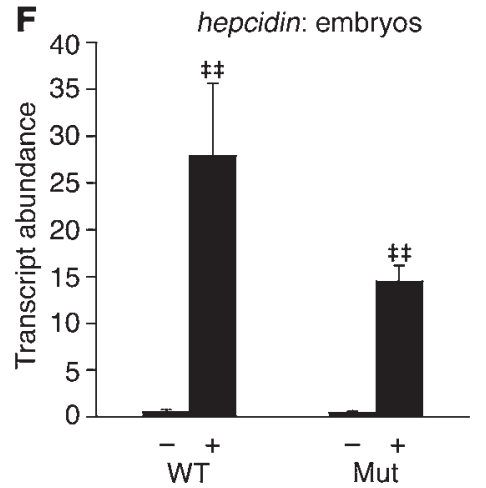

\section{Figure 6}

Quantification of transcript levels by multiplex real-time PCR. RNA from liver (A, B, and D) or intestine (C) of 10- to 12-month-old adult zebrafish or pools of embryos ( $E$ and $\mathbf{F}$ ) was used to generate cDNA templates for multiplex reactions amplifying the gene of interest and $\beta$-actin. Transcript abundance, normalized to $\beta$-actin expression, is expressed as a fold increase over a calibrator sample. (A-D) Expression of transferrin (A), fpn1 (B and C), or hepcidin (D) in adult WT, heterozygote, or mutant zebrafish treated with iron 6-8 months previously (+Fe) or never treated (no Fe). The calibrator sample was an uninjected WT adult; $n=6-9$ individuals per cohort. ${ }^{*} P=0.01$ compared with iron-injected WT zebrafish. ${ }^{*} P<0.0001$ compared with iron-injected WT zebrafish. (E and F) At 48 hours after fertilization, embryos were anesthetized and injected with iron dextran (+) or anesthetized without iron injection (-). (E) Eighteen-hour time course of hepcidin transcript abundance following iron dextran injection. The calibrator sample consisted of a pool of embryos 2 hours after iron injection; $n=2$ pools of embryos per cohort. $\ddagger P<0.05$ compared with uninjected

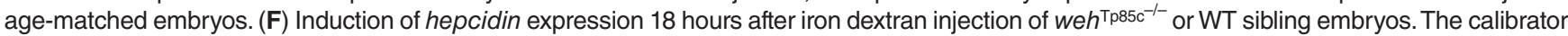
sample was an uninjected pool of embryos; $n=3$ pools of embryos per cohort. $¥ \neq P<0.01$ compared with uninjected age-matched embryos.

\section{Discussion}

The zebrafish mutant web is the first known animal model of fpn1 deficiency. Previous work demonstrated embryonic anemia in weh zebrafish attributed to a defect in iron transfer across the yolk syncytial layer (4). We have now demonstrated nonheme iron accumulation in the intestinal epithelium of $\mathrm{weh}^{\mathrm{Tp} 85 \mathrm{c}^{-/-}}$adults, consistent with a block in intestinal iron export. This resembles the pattern of iron accumulation in the sex-linked anemia $($ sla $)$ mouse $(27,28)$, which has a defect in intestinal iron export due to a mutation in the basolateral ferroxidase, hephaestin (29).

We propose that injecting eeb $^{\mathrm{Tp}} 85 \mathrm{c}^{-/-}$homozygotes with iron dextran rescues the animals by bypassing the block in intestinal iron absorption. Although most iron is tightly bound to dextran and would be phagocytosed by macrophages, approximately $2 \%$ is

\section{Figure 7}

Radioactive in situ hybridization for fpn1. (A) Iron-injected $w e h^{\mathrm{Tp} 85 c^{-/-}}$ zebrafish with increased $f p n 1$ expression compared with (B) iron-injected WT. Shown are intestines from zebrafish sacrificed at 1 year of age, fixed in $4 \%$ paraformaldehyde, embedded in paraffin, and hybridized with ${ }^{35}$ S-labeled antisense (left) or sense (right) probe for fpn1. Magnification, $\times 4$. Scale bar: $1 \mathrm{~mm}$. labile and can immediately bind transferrin (30) without further processing by the reticuloendothelial system. The increase in hepcidin expression observed in both WT and mutant embryos 18 hours after iron dextran injection supports the hypothesis that serum iron levels rise following the injection. Transferrin-bound iron would be readily delivered to the erythroid compartment, driving

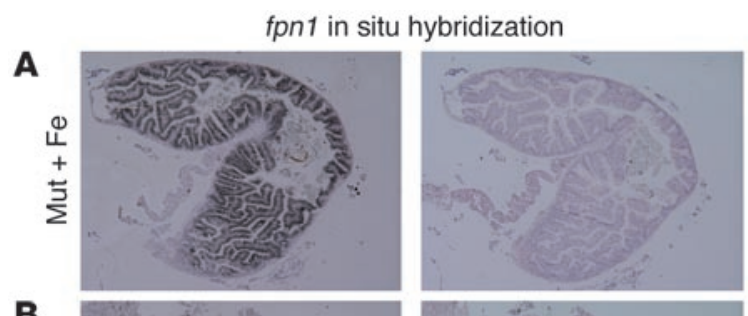

B

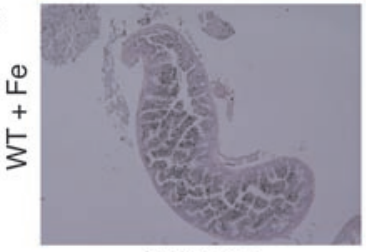

Antisense

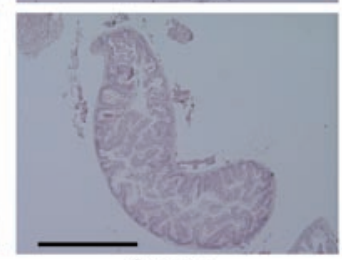

Sense 
hemoglobin synthesis and erythroid maturation. Over time, aging erythrocytes are phagocytosed by macrophages. The defect in fpn 1 function impairs iron export, resulting in retention of iron in the reticuloendothelial system and the onset of anemia. The anemia is associated with decreased transcript levels of the iron regulator hepcidin in web $^{\mathrm{Tp} 85 c^{-/-}}$zebrafish, while transferrin and hepatic fpn1 expression are not significantly different from that in controls.

Our analysis of fpn1-deficiency in weh $^{\mathrm{Tp} 85 \mathrm{c}^{-/-}}$zebrafish supports the critical role of fpn 1 in releasing iron to developing erythroid cells and is consistent with findings in the polycythemia ( $p c m$ ) mouse (31), which develops $p c m$ due to a hypermorphic allele of fpn 1 that lacks iron/ iron regulatory element (IRE)-mediated translational regulation due to a deletion in the promoter. At 7 weeks of age, pcm heterozygous mice with low hepcidin expression exhibit increased hepatic and duodenal fpn1 expression, expanded erythroid differentiation (31), and resulting polycythemia. On the other hand, our data illustrate that impaired fpn 1 function in $w_{e} b^{\mathrm{Tp} 85 c^{-/-}}$zebrafish is associated with hypochromic anemia and impaired erythroid maturation.

The decreased hepcidin expression demonstrated in anemic weh $^{\mathrm{Tp} 85 \mathrm{c}^{-/-}}$ adults and the increased levels in WT zebrafish and mutants following acute iron dextran injection provide the first evidence for a conserved role for hepcidin in the zebrafish as a regulator of iron homeostasis. Others have identified increases in hepcidin expression in response to bacterial infection in zebrafish (11), suggesting that hepcidin in zebrafish, as in other organisms, is part of the inflammatory response. The low hepcidin expression in anemic web $^{\mathrm{Tp} 85 c^{-/-}}$adults is consistent with the response to anemia observed in the hypotransferrinemic mouse, which also exhibits anemia despite adequate iron stores (32). Similarly, the Belgrade b/b rat, which has hypochromic anemia due to a mutation in DMT1, exhibits undetectable levels of hepcidin mRNA accompanied by an increase in intestinal but not hepatic fpn1 expression (33). Investigators have noted that administration of recombinant hepcidin peptide to rats results in a decline in duodenal but not hepatic fpn1 expression (33). We observed differences in hepatic and intestinal fpn1 expression that may reflect the effects of decreased hepcidin: mean intestinal $f p n 1$ transcript levels were increased in anemic $w_{e} h^{\mathrm{Tp} 85 c^{-/-}}$adults, but mean hepatic fpn1 mRNA levels were not significantly different from those of controls.

In addition to having effects on transcriptional regulation, hepcidin appears to be a critical homeostatic regulator affecting both iron uptake and iron export. Exposing cultured human intestinal epithelial cells to hepcidin (34) resulted in decreased apical iron uptake and diminished mRNA and protein expression of DMT1 ( 3 '-IRE-containing isoform). In addition, treatment of tissue culture cells expressing fpn1-GFP with hepcidin resulted in internalization and degradation of fpn 1 as well as decreased iron export (35). Further supporting a homeostatic loop involving fpn 1 and hepcidin, $p c m$ heterozygote mice with increased fpn 1 protein expression at 7 weeks developed increased hepcidin expression and a decline in fpn 1 protein to normal levels by 12 weeks (31), resulting in resolution of the polycythemia phenotype. The profoundly low hepcidin expression in $\mathrm{weh}^{\mathrm{Tp} 85 c^{-/-}}$zebrafish can also be seen as a homeostatic response to anemia. Unlike the pcm mouse, however, $w_{e} h^{\mathrm{Tp} 85 c^{-/-}}$zebrafish possess a missense mutation in fpn 1 , which results in a failure to correct the phenotype despite the compensatory change in hepcidin expression.

Given the homeostatic relationship of hepcidin and fpn 1 , it is conceivable that the $w_{e} b^{\mathrm{T} p 85 c}$ mutation might restrict bepcidin expression. Mice deficient in the hereditary hemochromatosis gene product (HFE) exhibit inappropriately low levels of hepcidin expression when fed a high-iron diet (36) or injected with iron dextran (37). These findings imply that HFE function is required for proper regulation of hepcidin expression in response to iron loading. In contrast, $w e b^{\mathrm{Tp} 85 c^{-/-}}$embryos injected with iron dextran manifest an appropriate increase in hepcidin expression, indicating that the L167F mutation in fpn 1 does not impair iron-induced regulation of hepcidin expression.

While the majority of hereditary hemochromatosis cases are attributed to mutations in the atypical MHC class I-protein HFE (38), it is now recognized that hereditary hemochromatosis can be associated with defects in hepcidin or hemojuvelin (type 2), transferrin receptor 2 (type 3), or fpn 1 (type 4) (39). The Tp85c allele of weh has a mutation (L167F) (4) at a conserved leucine in a loop between the third and fourth predicted transmembrane helices of fpn1 (22). A cluster of human mutations (Supplemental Table 1; available online with this article; doi:10.1172/JCI23780DS1) in patients with type 4 hemochromatosis or elevated serum ferritin has now been identified at conserved residues in this loop (20-26), suggesting that this may be a functional domain, and at other locations (24, 40-42) of fpn1. Although hepcidin expression has been found to be low in patients with hemochromatosis attributed to mutations in HFE (43), transferrin receptor 2 (44), hepcidin (45), or hemojuvelin (HFE2) (46), urinary protein levels have been found to be increased (47) in 2 hemochromatosis patients heterozygous for the Val162del mutation, localized between transmembrane helices 3 and 4 of fpn 1 . This is consistent with our observation that iron-responsive hepcidin expression is not ablated by the zebrafish L167F mutation. Taken together, these findings suggest that regulation of hepcidin expression is independent of fpn 1 function as an iron transporter.

While a gain of function mutation has not been ruled out in patients with type 4 hemochromatosis, the disease manifests with distinctive clinical features that are consistent with impaired iron export from the reticuloendothelial system. These features include accumulation of iron specifically in macrophages and Kupffer cells, absence of hepatic fibrosis, and a tendency among those who have the disease to develop anemia $(39,40)$ following venesection. Patients with type 4 hemochromatosis, unlike those with types 1 , 2 , or 3 , usually have high serum ferritin levels that appear to be disproportionate to their serum iron levels and transferrin saturations $(22,24)$. Although patients with type 4 hemochromatosis are heterozygous for $f p n 1$ missense mutations, we have not identified anemia or iron accumulation in $w e b^{\mathrm{T} p 85 c}$ heterozygotes. This raises the possibility that the effect of the zebrafish mutation is less severe than that of the human mutation or that there may be secondary mutations in fpn 1 or other modifying genes that contribute to iron overload. The presence or absence of a haploinsufficiency phenotype in zebrafish varies depending on the nature of the mutation: a mild anemia phenotype has been detected in zebrafish that are heterozygous for defects in solute carrier family 4 but not in the interacting erythrocyte membrane protein band 4.1 (48). The absence of a haploinsufficiency phenotype in $w e b^{\mathrm{T}} 85 \mathrm{c}$ zebrafish may be due to partial function of the mutated fpn 1 or to compensation by an alternative exporter.

In summary, we $b^{\mathrm{Tp} 85 c^{-/-}}$zebrafish have impaired function of fpn1, which results in defective intestinal and macrophage iron export. We have identified hepcidin as an iron-responsive gene in the zebrafish that does not require normal fpn 1 export function for its expression. Our studies demonstrate the use of zebrafish to study the interaction of genes involved in hemochromatosis and establish a foundation for further genetic analysis. 


\section{Methods}

Zebrafish strains, maintenance, and determination of genotype. Zebrafish were maintained as described (49). Ethical approval for these experiments was obtained from the Institutional Animal Care and Use Committees of Children's Hospital and Beth Israel Deaconess Medical Center. Web ${ }^{\mathrm{T} p 85 c}$ were maintained on the $\mathrm{AB}$ background. Homozygote and heterozygote progeny were generated by crosses of heterozygotes with heterozygotes or iron-rescued homozygotes. We ${ }^{\mathrm{Tp}} 85 \mathrm{c}^{+/+}$(WT) controls were siblings of the homozygotes generated by heterozygote incrosses. Additional WT zebrafish were generated in AB WT incrosses maintained in parallel. The embryos were assessed for anemia at 48 hours after fertilization. Embryos in the injected cohorts were first microinjected with $100 \mathrm{mg} / \mathrm{ml}$ iron dextran (SigmaAldrich) at 48-72 hours after fertilization, as described (4). Additional injections of iron dextran were performed i.m. at 3 weeks ( $4 \mathrm{nl}), 8$ weeks $(200 \mu \mathrm{l})$, and 16 weeks $(200 \mu \mathrm{l})$. Fish were of similar sizes across the cohorts, and dose was not adjusted for weight. Both injected and uninjected cohorts were fed an iron-rich diet of brine shrimp. Genotypes were confirmed by amplifying with the following primers: 5'-AATGGTCATCTCCATTGCTAATATCGC-3' and $5^{\prime}$-AAATGTACAATGGCCAAAAAACGAATT $-3^{\prime}$. The PCR product was purified using the QIAquick spin column (QIAGEN). The presence or absence of the mutation was determined by direct automated sequencing with the primer 5'-GCAATGAACTATAAATGG-3'.

Analysis of peripheral blood and kidney marrow. The peripheral blood was collected from individual adult zebrafish as previously described $(12,13)$ and washed in $40 \mathrm{ml} 0.9 \times$ PBS-1\% BSA. After centrifugation, the blood was resuspended in $200 \mu \mathrm{l}$ of $0.9 \times$ PBS-1\% BSA and analyzed on an automated cell counter (ADVIA 120; Bayer Diagnostics) for determination of MCV and CH.

MCV readings obtained from the ADVIA were validated by determining packed erythrocyte cell volume for WT zebrafish using classical techniques. Blood was pooled from cohorts of WT adult zebrafish ( $n=3$ cohorts, 9 fish per cohort). Pooled blood was washed, as described above, and resuspended in $60 \mu \mathrm{l}$ of $0.9 \times$ PBS-1\% BSA. The blood was then centrifuged in $9-\mu 1$ microcapillary tubes for 2 minutes in a StatSpin multipurpose centrifuge to determine the spun hematocrit. From the same samples, the cell density was determined by enumeration with a hemocytometer. The packed erythrocyte cell volume was calculated as follows: (spun hematocrit $\times 0.01 \times 10^{9} \mathrm{fl} / \mu \mathrm{l}$ )/ number of cells per $\mu$. Aliquots of the pooled blood samples were also diluted 10 -fold in $0.9 \times$ PBS-1\% BSA for evaluation of MCV on the ADVIA. We determined that the 2 techniques did not result in significantly different MCVs for WT zebrafish: $97.0 \pm 3.69 \mathrm{fl}$ (classical method) compared with $92.9 \pm 2.99 \mathrm{fl}$ obtained with the ADVIA $120(P=0.43)$. Data reported in our analyses were obtained on the ADVIA 120.

Kidney marrow was dissected and gently suspended in $0.5 \mathrm{ml}$ ice-cold PBS-5\% fetal calf serum containing heparin $(5 \mathrm{U} / \mathrm{ml})$. The samples were filtered through a 40 micron filter. Propidium iodide was added to a concentration of $1 \mu \mathrm{g} / \mathrm{ml}$ to exclude dead cells. As previously described (48), $1 \times 10^{5}$ cells were analyzed for each fish by flow cytometry, using differences in FSC and SSC to obtain automated differentials. Cell populations were analyzed with the program FlowJo 4.6 (Treestar Inc.). Cytospins of peripheral blood and kidney marrow were also obtained from $1 \times 10^{5}$ kidney cells using a Shandon Cytospin 3 centrifuge (Thermo Electron Corp.). Cytospin slides were processed through Wright and Giemsa stains (Harleco) for morphological analysis and cell counts.

In situ bybridization and histological analysis. Zebrafish tissues were fixed in $4 \%$ paraformaldehyde- $1 \times \mathrm{PBS}\left(\mathrm{pH} 7.4\right.$ ) at $4^{\circ} \mathrm{C}$ for 24 hours, embedded in paraffin, and sectioned at a thickness of 4 microns. As riboprobes $0.5-1 \mathrm{~kb}$ in size are optimal for in situ hybridization on fixed sections, cDNA templates for the riboprobes were generated by amplifying smaller pieces within the open reading frame of $f p n 1$. Sp 6 and T7 were incorporated into the forward and reverse primers, respectively, for subsequent run-off. The
PCR reaction conditions were as follows: $25 \mathrm{ng}$ template (cloned full-length cDNA provided by A. Donovan), $0.2 \mathrm{mM}$ dNTP, $0.2 \mu \mathrm{M}$ forward primer, $0.2 \mu \mathrm{M}$ reverse primer, $1 \times$ Advantage 2 Polymerase Mix (Invitrogen Corp.). Cycling was as follows: $94^{\circ} \mathrm{C}$ for 2 minutes, followed by 35 cycles at $94^{\circ} \mathrm{C}$ for 20 seconds, $55^{\circ} \mathrm{C}$ for 30 seconds, and $68^{\circ} \mathrm{C}$ for 3 minutes, followed by $68^{\circ} \mathrm{C}$ for 10 minutes. Primers were as follows: Fpn1F3Sp6 (5'-CAAGCTTGATTTAGGTGACACTATAGAATACAATGCAAAATTCCTCATTTACGTCG-3'), Fpn1R9T7 (5'-GTGAATTGTAATACGACTCACTATAGGGCGCTGCCAATTTGCTCCGATCAT-3') to generate a $500 \mathrm{bp}$ fragment (nucleotides 763-1193). The size of the PCR products was verified by electrophoresis. The PCR reactions were purified with a QIAquick spin column and brought to a final concentration of $1 \mu \mathrm{g} / \mu \mathrm{l}$. $\mathrm{S}^{35}$-labeled riboprobes were prepared with an in vitro transcription kit (Roche Diagnostics) using T7 or Sp6 to run off the antisense or sense probe, respectively. The efficiency of transcription and incorporation of $\mathrm{S}^{35}$-UTP into the riboprobes was evaluated by $5 \%$ acrylamide-urea gel electrophoresis and scintillation counting.

Radioactive in situ hybridization was performed on paraffin-embedded sections mounted on slides. After deparaffinization, slides were fixed in $4 \%$ paraformaldehyde for 10 minutes and digested with proteinase $\mathrm{K}(10 \mu \mathrm{g} / \mathrm{ml})$ for 10 minutes at $37^{\circ} \mathrm{C}$. Sense and antisense riboprobes were diluted in hybridization buffer to $2 \times 10^{6}$ counts per minute per slide. Hybridization was performed overnight at $60^{\circ} \mathrm{C}$. After hybridization, slides were washed at $65^{\circ} \mathrm{C}$ for 2 hours in $0.1 \times$ SSC. Slides were dipped in Kodak NTB2 emulsion, exposed at $4^{\circ} \mathrm{C}$ for 4 weeks, developed, and counterstained with $\mathrm{H} \& \mathrm{E}$. Corresponding sections from the same animals were stained either with H\&E or Perls' stain. For Perls' stain $(50,51)$, sections were deparaffinized, incubated in $5 \%$ potassium ferrocyanide $/ 5 \% \mathrm{HCl}$ for 20 minutes, washed in distilled water, then counterstained with nuclear fast red. DAB-enhanced iron staining was performed as follows: following deparaffinization, the slides were incubated with $1 \%$ potassium ferrocyanide $/ 0.12 \mathrm{~N} \mathrm{HCl}$, washed with PBS, quenched in $0.3 \% \mathrm{H}_{2} \mathrm{O}_{2}$ in methanol for 20 minutes, washed in PBS, and incubated in DAB $/ \mathrm{H}_{2} \mathrm{O}_{2}$ (DAB kit no. 2020; Zymed Laboratories) for 7 minutes. No counterstain was performed on DAB-stained sections.

Nonheme iron quantification. Male zebrafish were sacrificed to avoid potential contamination of samples from iron-rich oocytes. Livers were dissected, pooled, and weighed in groups of 6 (at 8 months) or 3 (at 12 months) and lysed for 48 hours at $60^{\circ} \mathrm{C}$ in $250 \mu \mathrm{l}$ of $3 \mathrm{M}$ hydrochloric acid/0.61 M trichloroacetic acid in iron-free water. One $\mathrm{ml}$ of chromagen solution ( $0.01 \%$ bathophenanthroline sulfonate, $0.1 \%$ thioglycolic acid, saturated sodium acetate) was added to $50 \mu \mathrm{l}$ of lysate. OD was measured at $535 \mathrm{~nm}$ according to the method previously described (52). Iron was quantified in comparison to a standard curve generated from iron standards (no. 565-11; Sigma-Aldrich). The values were expressed as $\mu \mathrm{g}$ iron/g of wet tissue.

Quantitative analysis of gene expression. Individual web ${ }^{\mathrm{T} p 55 c}$ mutant and control zebrafish were dissected to obtain liver and intestine. Dissected organs were immediately placed in RNAlater (Ambion) to stabilize transcripts. To analyze the effects of iron injection on embryonic gene expression, 48-hour-old embryos were injected with iron dextran or anesthetized. At specified time points after treatment, embryos were pooled in groups of 18-20, euthanized with tricaine, and placed in RNAlater. Each organ or embryo pool was frozen with liquid nitrogen and ground with a mortar and pestle. RNA was then extracted from the ground tissue using the RNeasy lysis and purification procedure (QIAGEN). RNA yield was quantified by absorption at $260 \mathrm{~nm}$. To obtain cDNA, a reverse transcription was performed on $100 \mathrm{ng}$ of RNA (for organs) or 1 pool (for embryos) using Superscript II reverse transcriptase (Invitrogen Corp.) in a $20-\mu \mathrm{l}$ reaction. The reaction was then diluted to $100 \mu \mathrm{l}$. For each real-time PCR reaction, $10 \mu \mathrm{l}$ were used as template. Reaction cocktail total volume was $50 \mu \mathrm{l}$ and included $0.4-\mu \mathrm{M}$ forward primer, $0.4-\mu \mathrm{M}$ reverse primer, $0.2-\mu \mathrm{M}$ probe, and $1 \times$ QuantiTect Probe PCR Master Mix (QIAGEN). Reactions were performed in duplicate. Cycling parameters were 
as follows: $50^{\circ} \mathrm{C}$ for 2 minutes and $95^{\circ} \mathrm{C}$ for 15 minutes, followed by 40 cycles at $94^{\circ} \mathrm{C}$ for 15 seconds and $60^{\circ} \mathrm{C}$ for 1 minute. Detection and analysis were performed on an ABI Prism 7700 (Applied Biosystems).

Each reaction was designed to quantitate expression of $f p n 1$, transferrin, or hepcidin, normalized to zebrafish $\beta$-actin, according to a previously described method (53). Transcript abundance was expressed as a fold increase over calibrator sample. To design the primers used in the real-time PCR assays, sequences for the zebrafish genes of interest ( $f p n 1$, transferrin, and hepcidin) as compiled in Genbank were blasted against the zebrafish genome assembly to identify predicted intron-exon boundaries. The $\beta$-actin primer probe set was as previously described (53). The genes of interest were amplified with the following primers, each spanning a predicted intron-exon boundary: $f p n 1$, 5' - GGCCAGCACAGCTATGTC-3', 5'-GCCAGAATGTTGGTCAACTG-3'; transferrin, 5' - TTACATGGGAGGGTCCTAATGAG-3', 5'- GGACACAACTGCTCGAGAAGAA-3'; hepcidin, 5'- CCTGGCTGCTGTCGTCAT-3', 5'- TGGTTCTCCTGCAGTTCTTCAC-3'; and $\beta$-actin, 5'- AgGtCATCACCATCGgCAAT-3', 5'- GAtgtCCACGTCGCACTTCAT- $3^{\prime}$. Probes were as follows: $f p n 1,5^{\prime}$-FAM-TGCATTCATATCTGCCAATTTGCTCCGA-TAMRA-3'; transferrin, 5'-FAMCTGACACAGCCCTCTCGACAGG-TAMRA-3'; hepcidin, 5'-FAMCCGTTCCCTTCATACAGCAGGTACAGG-TAMRA-3'; and $\beta$-actin, $5^{\prime}$-VICCTTCCAGCCTTCCTTCCTGGGTATGGA-TAMRA-3'.

The $100 \mathrm{bp}$ amplicons for each gene were cloned and sequenced to confirm amplification of the appropriate genes. Serial dilutions of the cloned amplicons were tested in the assay. Cycle thresholds were plotted versus natural log of template concentration, yielding linear results over a range of template concentrations from $1.4 \times 10^{-17}$ to $1.4 \times 10^{-12} \mathrm{M}$. The coefficients of variation determined by linear regression were -0.9837 ( $\beta$-actin), -0.9887 (fpn1), -0.9801 (hepcidin), and -0.9974 (transferrin). In each assay of adult organs, cDNA from the same uninjected WT zebrafish was used as a calibrator template. For pooled embryos, the calibrator template was 1 of the age-matched embryo control pools. The reactions failed to amplify nontemplate controls, genomic DNA, or RNA preparations that were not treated with reverse transcriptase.
Biostatistical analysis. Heterogeneity among age-matched cohorts was analyzed by ANOVA. Results reported from the ANOVA analysis are means $\pm \mathrm{SE}$. Tests for heterogeneity among age-matched cohorts used the natural logarithm for the assessment of nonheme iron levels and fpn1, transferrin, and hepcidin transcript levels. All estimates and standard errors presented have been transformed back to the original units. When the global $P$ value obtained from the ANOVA analysis was statistically significant, pairwise comparisons between the age-matched cohorts were performed. All $P$ values were obtained from 2 -sided Student's $t$ tests. We did not adjust for multiple comparisons. $P$ values less than or equal to 0.05 were deemed statistically significant. ANOVA and Student's $t$ tests were performed using InStat 3.0 (GraphPad Software).

\section{Acknowledgments}

We thank Victoria Petkova of the Beth Israel Deaconess Medical Center Real-Time PCR Core for assistance with the real-time PCR assay and Yu Yang of the Harvard Dana-Farber Cancer Institute In Situ Hybridization Core Laboratory for assistance with in situ hybridizations. We are grateful to Tenora Archibald, Children's Hospital, for assistance with histopathology. We also thank Kim Dooley and Rebecca Wingert for critical review of the manuscript and Mark Fleming for fruitful discussions. This work was supported by NIH grants DK-61685-03 (to P.G. Fraenkel) and DK-053298-07 (to L.I. Zon), the Howard Hughes Medical Institute grant (to L.I. Zon), and the American Society of Hematology Scholar Award (P.G. Fraenkel).

Received for publication October 29, 2004, and accepted in revised form March 29, 2005.

Address correspondence to: L.I. Zon, Division of Hematology/ Oncology, Children's Hospital, Karp Research Laboratories, 1 Blackfan Circle, Boston, Massachusetts 02115, USA. Phone: (617) 9192069; Fax: (617) 730-0222; E-mail: zon@enders.tch.harvard.edu.
1. Hentze, M.W., Muckenthaler, M.U., and Andrews, N.C. 2004. Balancing acts: molecular control of mammalian iron metabolism. Cell. 117:285-297.

2. Andrews, N.C. 1999. The iron transporter DMT1 Int. J. Biochem. Cell Biol. 31:991-994.

3. Andrews, N.C., Fleming, M.D., and Levy, J.E. 1999. Molecular insights into mechanisms of iron transport. Curr. Opin. Hematol. 6:61-64.

4. Donovan, A., et al. 2000. Positional cloning of zebrafish ferroportin 1 identifies a conserved vertebrate iron exporter. Nature. 403:776-781.

5. Park, C.H., Valore, E.V., Waring, A.J., and Ganz, T 2001. Hepcidin, a urinary antimicrobial peptide synthesized in the liver. J. Biol. Chem. 276:7806-7810.

6. Krause, A., et al. 2000. LEAP-1, a novel highly disulfide-bonded human peptide, exhibits antimicrobial activity. FEBS Lett. 480:147-150.

7. Nicolas, G., et al. 2001. Lack of hepcidin gene expression and severe tissue iron overload in upstream stimulatory factor 2 (USF2) knockout mice. Proc. Natl. Acad. Sci. U. S. A. 98:8780-8785.

8. Nicolas, G., et al. 2002. Severe iron deficiency anemia in transgenic mice expressing liver hepcidin. Proc. Natl. Acad. Sci. U. S. A. 99:4596-4601.

9. Nicolas, G., et al. 2002. The gene encoding the iron regulatory peptide hepcidin is regulated by anemia, hypoxia, and inflammation. J. Clin. Invest. 110:1037-1044. doi:10.1172/JCI200215686.

10. Pigeon, C., et al. 2001. A new mouse liver-specific gene, encoding a protein homologous to human antimicrobial peptide hepcidin, is overexpressed during iron overload. J. Biol. Chem. 276:7811-7819.
11. Shike, H., Shimizu, C., Lauth, X., and Burns, J.C. 2004. Organization and expression analysis of the zebrafish hepcidin gene, an antimicrobial peptide gene conserved among vertebrates. Dev. Comp. Immunol. 28:747-754.

12. Brownlie, A., et al. 2003. Characterization of embryonic globin genes of the zebrafish. Dev. Biol. 255:48-61.

13. Brownlie, A., et al. 1998. Positional cloning of the zebrafish sauternes gene: a model for congenital sideroblastic anaemia. Nat. Genet. 20:244-250.

14. Donovan, A., et al. 2002. The zebrafish mutant gene chardonnay (cdy) encodes divalent metal transporter 1 (DMT1). Blood. 100:4655-4659.

15. Wingert, R.A., et al. 2004. The chianti zebrafish mutant provides a model for erythroid-specific disruption of transferrin receptor 1. Development. 131:6225-6235.

16. McKie, A.T., et al. 2000. A novel duodenal ironregulated transporter, IREG1, implicated in the basolateral transfer of iron to the circulation. Mol. Cell. 5:299-309.

17. Abboud, S., and Haile, D.J. 2000. A novel mammalian iron-regulated protein involved in intracellular iron metabolism. J. Biol. Chem. 275:19906-19912.

18. Lymboussaki, A., et al. 2003. The role of the iron responsive element in the control of ferroportin1/IREG1/MTP1 gene expression. J. Hepatol. 39:710-715.

19. Ransom, D.G., et al. 1996. Characterization of zebrafish mutants with defects in embryonic hematopoiesis. Development. 123:311-319.
20. Wallace, D.F., et al. 2002. Novel mutation in ferroportin 1 is associated with autosomal dominant hemochromatosis. Blood. 100:692-694.

21. Roetto, A., et al. 2002. A valine deletion of ferroportin 1: a common mutation in hemochromastosis type 4. Blood. 100:733-734.

22. Devalia, V., et al. 2002. Autosomal dominant reticuloendothelial iron overload associated with a 3-base pair deletion in the ferroportin 1 gene (SLC11A3). Blood. 100:695-697.

23. Njajou, O.T., et al. 2001. A mutation in SLC11A3 is associated with autosomal dominant hemochromatosis. Nat. Genet. 28:213-214.

24. Hetet, G., Devaux, I., Soufir, N., Grandchamp, B., and Beaumont, C. 2003. Molecular analyses of patients with hyperferritinemia and normal serum iron values reveal both $L$ ferritin IRE and 3 new ferroportin (slc11A3) mutations. Blood. 102:1904-1910.

25. Arden, K.E., et al. 2003. A novel mutation in ferroportin 1 is associated with haemochromatosis in a Solomon Islands patient. Gut. 52:1215-1217.

26. Wallace, D.F., Clark, R.M., Harley, H.A., and Subramaniam, V.N. 2004. Autosomal dominant iron overload due to a novel mutation of ferroportin 1 associated with parenchymal iron loading and cirrhosis. J. Hepatol. 40:710-713.

27. Edwards, J.A., and Bannerman, R.M. 1970. Hereditary defect of intestinal iron transport in mice with sex-linked anemia. J. Clin. Invest. 49:1869-1871.

28. Manis, J. 1971. Intestinal iron-transport defect in the mouse with sex-linked anemia. Am J. Physiol. 220:135-139. 
29. Vulpe, C.D., et al. 1999. Hephaestin, a ceruloplasmin homologue implicated in intestinal iron transport, is defective in the sla mouse. Nat. Genet. 21:195-199.

30. Van Wyck, D., Anderson, J., and Johnson, K. 2004. Labile iron in parenteral iron formulations: a quantitative and comparative study. Nephrol. Dial. Transplant. 19:561-565.

31. Mok, H., et al. 2004. Disruption of ferroportin 1 regulation causes dynamic alterations in iron homeostasis and erythropoiesis in polycythaemia mice. Development. 131:1859-1868.

32. Weinstein, D.A., et al. 2002. Inappropriate expression of hepcidin is associated with iron refractory anemia: implications for the anemia of chronic disease. Blood. 100:3776-3781.

33. Yeh, K.Y., Yeh, M., and Glass, J. 2004. Hepcidin regulation of ferroportin 1 expression in the liver and intestine of the rat. Am. J. Physiol. Gastrointest. Liver Physiol. 286:G385-G394.

34. Yamaji, S., Sharp, P., Ramesh, B., and Srai, S.K. 2004. Inhibition of iron transport across human intestinal epithelial cells by hepcidin. Blood. 104:2178-2180.

35. Nemeth, E., et al. 2004. Hepcidin regulates cellular iron efflux by binding to ferroportin and inducing its internalization. Science. 306:2090-2093.

36. Ahmad, K.A., et al. 2002. Decreased liver hepcidin expression in the Hfe knockout mouse. Blood Cells
Mol. Dis. 29:361-366.

37. Muckenthaler, M., et al. 2003. Regulatory defects in liver and intestine implicate abnormal hepcidin and Cybrd 1 expression in mouse hemochromatosis. Nat. Genet. 34:102-107.

38. Merryweather-Clarke, A.T., Pointon, J.J., Jouanolle, A.M., Rochette, J., and Robson, K.J. 2000. Geography of HFE C282Y and H63D mutations. Genet. Test. 4:183-198.

39. Pietrangelo, A. 2004. Hereditary hemochromatosis-a new look at an old disease. N. Engl. J. Med. 350:2383-2397.

40. Montosi, G., et al. 2001. Autosomal-dominant hemochromatosis is associated with a mutation in the ferroportin (SLC11A3) gene. J. Clin. Invest. 108:619-623. doi:10.1172/JCI200113468.

41. Gordeuk, V.R., et al. 2003. Iron overload in Africans and African-Americans and a common mutation in the SCL40A1 (ferroportin 1) gene. Blood Cells Mol. Dis. 31:299-304.

42. Jouanolle, A.M., et al. 2003. Novel mutation in ferroportin 1 gene is associated with autosomal dominant iron overload. J. Hepatol. 39:286-289.

43. Bridle, K.R., et al. 2003. Disrupted hepcidin regulation in HFE-associated haemochromatosis and the liver as a regulator of body iron homoeostasis. Lancet. 361:669-673.

44. Nemeth, E., Roetto, A., Garozzo, G., Ganz, T., and Camaschella, C. 2005. Hepcidin is decreased in
TFR2 hemochromatosis. Blood. 105:1803-1806.

45. Roetto, A., et al. 2003. Mutant antimicrobial peptide hepcidin is associated with severe juvenile hemochromatosis. Nat. Genet. 33:21-22.

46. Papanikolaou, G., et al. 2004. Mutations in HFE2 cause iron overload in chromosome $1 \mathrm{q}$-linked juvenile hemochromatosis. Nat. Genet. 36:77-82.

47. Papanikolaou, G., et al. 2005. Hepcidin in iron overload disorders. Blood. doi:10.1182/blood-200412-4844.

48. Traver, D., et al. 2003. Transplantation and in vivo imaging of multilineage engraftment in zebrafish bloodless mutants. Nat. Immunol. 4:1238-1246.

49. Westerfield, M. 1994. The zebrafish book: a guide for the laboratory use of zebrafish (Brachydanio rerio). University of Oregon Press. Eugene, Oregon, USA.

50. Sheehan, D.C., and Hrapchak, B.B. 1980. Theory and practice of histotechnology. Battelle Press. Columbus, Ohio, USA. 217-218.

51. Luna, L. 1968. Manual of histologic staining methods of the AFIP. McGraw-Hill. New York, New York, USA. 183 pp.

52. Cook, J.D. 1980. Methods in hematology. Churchill Livingstone. New York, New York, USA. 105-108.

53. Trant, J.M., Gavasso, S., Ackers, J., Chung, B.C., and Place, A.R. 2001. Developmental expression of cytochrome P450 aromatase genes (CYP19a and CYP19b) in zebrafish fry (Danio rerio). J. Exp. Zool. 290:475-483. 